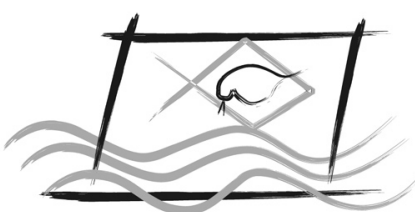

J. Braz. Soc. Ecotoxicol., v. 7, n. 1, 2012, 21-26

doi: $10.5132 / j b s e .2012 .01 .004$

ECOTOX - BRASIL

\title{
Alimento complementar adicionado às culturas de Daphnia similis e Ceriodaphnia dubia: efeitos da levedura e da digestão da ração
}

\author{
S.V. Buratini* \& M.A. Aragão \\ Companhia Ambiental do Estado de São Paulo - CETESB \\ Av. Prof. Frederico Hermann Jr, 345 - São Paulo - SP - Brasil.
}

(Received May 14, 2009; Accept May 20, 2010)

\begin{abstract}
Resumo
Durante uma década, a dieta constituída pela alga verde Pseudokirchneriella subcapitata e pelo alimento composto denominado RL (suspensão de ração para peixes digerida acrescida de levedura), foi utilizada no cultivo de dafinídeos, Daphnia similis e Ceriodaphnia dubia, pelo Laboratório de Ecotoxicologia Aquática da CETESB (Companhia Ambiental do Estado de São Paulo, SP, Brasil). Entretanto, oscilações na qualidade das culturas, com comprometimento da sobrevivência dos organismos adultos, foram associadas à adição do RL, o que conduziu à realização de experimentos nos quais o cultivo foi submetido ao fornecimento desse alimento complementar com e sem adição de levedura e, posteriormente, com inclusão de ração digerida e não digerida (ou solubilizada). Os resultados demonstraram que a complementação da dieta apenas com ração solubilizada e sem levedura permitiu o atendimento aos critérios de qualidade das culturas de Daphnia similis e Ceriodaphnia dubia, estabelecidos pelos respectivos procedimentos padronizados.
\end{abstract}

Palavras-chave: Daphnia similis; Ceriodaphnia dubia; cultivo; alimento composto; dieta.

Food supplement in Daphnia similis and Ceriodaphnia dubia cultures: effects of yeast and feed digestion

\begin{abstract}
Over ten years the diet consisting of green algae (Pseudokirchneriella subcapitata) and a suspension of digested fish flake food plus yeast was employed in culturing daphnids, Daphnia similis and Ceriodaphnia dubia in the Aquatic Ecotoxicology Laboratory of CETESB (Companhia Ambiental do Estado de São Paulo, SP, Brazil). However, oscillations in the quality of cultures, with adverse effects on adult survival, were attributed to the addition of this complement. For this reason, experiments were conducted to evaluate the supplying of such mixture with the presence and absence of yeast and, later on, with and without digestion of fish flake food. The results showed that a diet including algae and the suspension of non digested (solubilized) fish flake food, permitted the attainment of the quality criteria for Daphnia similis and Ceriodaphnia dubia cultures, established in the respective standard procedures.
\end{abstract}

Key words: Daphnia similis; Ceriodaphnia dubia; culture; diet; feeding suspension. 


\section{INTRODUÇÃO}

A qualidade nutricional do alimento fornecido às culturas de microcrustáceos da Família Daphniidae utilizados em ensaios ecotoxicológicos, pode determinar diferenças no seu desenvolvimento (especialmente sobre a longevidade e a reprodução) e na sua sensibilidade a agentes tóxicos (Lee et al., 1986; Baird et al., 1989) .

Os diferentes ensaios ecotoxicológicos padronizados com espécies de Daphnia e Ceriodaphnia recomendam uma dieta à base de um alimento natural constituído, preferencialmente, por uma alga clorofícea ou diatomácea e de um alimento complementar, representado por um ou mais dos seguintes componentes: ração de truta digerida, levedura, Cerophyll ${ }^{\circledR}$ (nome comercial dado à mistura de folhas de cereais desidratadas e trituradas) e Artemia (ENVIRONMENT CANADA, 1990; ASTM, 1997; USEPA, 2002a e 2002b). Da mesma forma, as normas nacionais correspondentes, ABNT-NBR 12713 (ABNT, 2004) e ABNT-NBR 13373 (ABNT, 2005) sugerem o fornecimento de algas e de um alimento complementar à base de ração para peixes digerida e levedura.

Tal complemento pode constituir uma fonte de variabilidade e produzir efeitos negativos intermitentes, tanto nas culturas como nos controles dos testes, uma vez que não têm sua composição definida e controlada - o grau de digestão da ração, por exemplo, depende muito da temperatura em que esta ocorre e a solução resultante pode ser bastante variável (Knight \& Waller, 1992; Bailey et al., 2000). Além disso, em ensaios crônicos, como o mesmo alimento é fornecido diariamente, este pode exercer interferências sobre a disponibilidade do agente químico considerado e, portanto, sobre o resultado do ensaio (Bradley et al., 1993). Por esse motivo, La Rocca et al. (1994) mencionam que a dieta dos organismos aquáticos usada em ensaios ecotoxicológicos deve ser considerada quando os resultados analíticos são comparados entre diferentes laboratórios.
No laboratório de Ecotoxicologia Aquática da CETESB, a dieta adotada desde 1991, constituída pela alga clorofícea Pseudokirchneriella subcapitata e pelo alimento composto de ração para peixes Tetramin ${ }^{\circledR}$ digerida e acrescida de levedura (denominado RL), foi utilizada com sucesso no cultivo de Daphnia similis e Ceriodaphnia dubia durante, aproximadamente, uma década. Entretanto, nesse período, freqüentemente ocorreram oscilações na qualidade das culturas, com comprometimento da sobrevivência dos organismos adultos, as quais foram atribuídas à redução dos níveis de oxigênio dissolvido devido à formação de uma película na superfície da água, provavelmente associada ao RL. Por essa razão, foram efetuados dois estudos com ambos os microcrustáceos: no primeiro verificou-se a necessidade de inclusão da levedura na dieta e no segundo avaliou-se o desempenho dos organismos quando fornecida ração digerida e ração solubilizada.

\section{MATERIAL E MÉTODOS}

\section{Dietas}

As dietas utilizadas nos experimentos foram preparadas conforme ABNT-NBR 12713 (ABNT, 2004) e ABNT-NBR 13373 (ABNT, 2005). Sua composição e as quantidades fornecidas às culturas encontram-se descritas na Tabela 1.

\section{EXPERIMENTOS}

Para avaliação do emprego da levedura, foram acompanhadas duas gerações de Daphnia similis e três gerações de Ceriodaphnia dubia. Foram preparadas 20 replicatas para cada microcrustáceo, contendo um organismo cada. Em dez replicatas adicionou-se suspensão algácea e ração digerida acrescida de levedura (RL); nas restantes adicionou-se suspensão algácea e ração digerida (RD). Já nos ensaios para

Tabela 1 - Composição e quantidades das dietas fornecidas nos experimentos com Daphnia similis e Ceriodaphnia dubia.

\begin{tabular}{|c|c|c|}
\hline Alimento & Composição & Quantidade fornecida/ organismo-teste \\
\hline \multirow[t]{2}{*}{ Suspensão algácea } & $\begin{array}{l}\text { Suspensão de Pseudokirchneriella subcapitata, na concentração de } \\
\qquad 3 \text { a } 3,5 \times 10^{7} \text { céls } / \mathrm{mL} .\end{array}$ & $3,2 \times 10^{6}$ céls/Daphnia/dia \\
\hline & & $1,6 \times 10^{5}$ céls/Ceriodaphnia/dia $(0,1 \mathrm{~mL})$ \\
\hline $\begin{array}{l}\text { RD } \\
\text { (Ração para peixes } \\
\text { digerida) }\end{array}$ & $\begin{array}{l}\text { Ração para peixes Tetramin }{ }^{\circledR} \text {, na proporção de } 10,0 \mathrm{gL}^{-1\left(^{(*)}\right.} \text { de água } \\
\text { desionizada, submetida à aeração durante } 7 \text { dias. Após esse período } \\
\text { a aeração é interrompida, o sobrenadante é coletado e filtrado em } \\
\text { rede de plâncton de malha } 60 \mu \mathrm{m} \text {. }\end{array}$ & $\begin{array}{c}0,05 \mathrm{~mL} / \text { Daphnia/dia } \\
0,05 \mathrm{~mL} / \text { Ceriodaphnia } / \text { dia }\end{array}$ \\
\hline $\begin{array}{l}\text { RL } \\
\text { (Ração para peixes } \\
\text { digerida } \\
\text { + levedura) }\end{array}$ & $\begin{array}{l}\text { Alimento formado pela adição de volumes iguais de solução de } \\
\text { ração para peixes Tetramin } \AA \text { (obtida a partir da digestão de } 5 \mathrm{~g} \\
\text { de ração em } 1 \mathrm{~L} \text { de água desionizada durante } 7 \text { dias) e solução } \\
\text { de levedura }\left(0,5 \mathrm{~g} \text { de fermento biológico granulado Fleishmann }{ }^{\circledR}\right. \\
\qquad / 100 \mathrm{~mL} \text { água desionizada). }\end{array}$ & $\begin{array}{c}0,05 \mathrm{~mL} / \text { Daphnia/dia } \\
0,05 \mathrm{~mL} / \text { Ceriodaphnia/dia }\end{array}$ \\
\hline $\begin{array}{l}\text { RS } \\
\text { (Ração para peixes } \\
\text { solubilizada) }\end{array}$ & $\begin{array}{l}\text { Ração para peixes Tetramin }{ }^{\circledR} \text {, na proporção de } 10 \mathrm{~g} \mathrm{~L}^{-1\left(^{(*)}\right.} \text { de água } \\
\text { desionizada, submetida à aeração vigorosa durante uma hora. Após } \\
\text { esse período, a aeração é interrompida por uma hora, quando o } \\
\text { sobrenadante é coletado e filtrado em rede de plâncton com malha } \\
\text { de } 60 \mu \mathrm{m} \text {. }\end{array}$ & $0,05 \mathrm{~mL} /$ Ceriodaphnia/dia \\
\hline
\end{tabular}

(*) A quantidade de ração foi dobrada de modo a manter o teor de sólidos totais dissolvidos previsto em ABNT (2004), ou seja, 2,5 a 3,1 g L-1. 
verificar a influência do processo de digestão da ração, foram acompanhadas oito gerações de cada microcrustáceo. Para cada uma das espécies foram utilizadas 40 replicatas, também com um organismo em cada, adicionando-se suspensão algácea e ração digerida (RD) em 20 replicatas e suspensão algácea e ração solubilizada (RS) nas outras 20.

Nos experimentos com Daphnia similis foram utilizados organismos jovens, obtidos segundo procedimento descrito em ABNT-NBR 12713 (ABNT, 2004). Em cada geração os indivíduos foram mantidos em meio MS (Keating, 1985) por um período de 21 dias, com alimentação diária e renovação da água três vezes por semana (ASTM, 1997; ISO, 2000). Foram considerados apropriados à manutenção deste microcrustáceo em laboratório os tratamentos que proporcionaram, no período de 21 dias, sobrevivência igual ou superior a $80 \%$ das adultas (limite adotado como indicador de culturas estoque saudáveis, em condições de fornecer jovens para a realização de ensaios, segundo ENVIRONMENT CANADA (1990) e ABNT (2004)) e reprodução igual ou superior a 60 jovens/ adulta (ASTM, 1997; ISO, 2000).

Os ensaios com Ceriodaphnia dubia foram iniciados com organismos jovens (6 a 30 horas de idade) e seguiram as mesmas condições descritas em ABNT-NBR 13373 (ABNT, 2005). No experimento para avaliação da levedura, os organismos foram mantidos em meio MS pelo período de 7 dias; já no estudo para avaliação da digestão da ração, cada uma das gerações de organismos foi mantida em água natural por 14 dias. Foram considerados adequados ao cultivo os tratamentos em que a sobrevivência dos organismos adultos ao final de 7 ou 14 dias manteve-se igual ou superior a $80 \%$, com produção de, no mínimo, 15 jovens/adulta (ABNT, 2005).

\section{ANÁLISE ESTATÍSTICA}

Em ambos os estudos, os resultados obtidos na geração $F_{0}$ foram excluídos das análises, uma vez que esta destinou-se à aclimatação dos organismos provenientes da condição controle ao tratamento em teste. Nas gerações seguintes avaliou-se a sobrevivência das adultas por meio do teste de Fischer; já os resultados relativos à reprodução foram, inicialmente, analisados quanto à normalidade (testes de Shapiro Wilks e quiquadrado) e homocedasticidade (teste F). Se atendidas essas condições, aplicou-se o teste " $\mathrm{t}$ " para Daphnia similis e " $\mathrm{t}$ " por bioequivalência para Ceriodaphnia dubia; quando não observada homogeneidade das variâncias aplicou-se o teste de Wilcoxon's Rank Sum. Todos esses procedimentos foram efetuados por meio do Programa TOXSTAT 3.5 (West Inc. \& Gulley, 1996).

\section{RESULTADOS E DISCUSSÃO}

\section{Efeitos da levedura}

$\mathrm{Na}$ dieta com inclusão de levedura (RL), observou-se uma variabilidade no desempenho da cultura de Daphnia similis, registrando-se em $F_{1}$ uma baixa sobrevivência dos organismos parentais (40\%) que não se confirmou na geração seguinte (Figura 1a). Ressalta-se que essa taxa de sobrevivência apresentou diferença estatística significativa em relação àquela obtida no tratamento com ração digerida, onde essa variável manteve-se estável e satisfatória (90 e $100 \%$ em $F_{1}$ e $F_{2}$, respectivamente). No que se refere à reprodução, no tratamento com adição de RL registrou-se um coeficiente de variação bastante elevado, visto que a baixa produtividade registrada em $\mathrm{F}_{1}$ (inferior ao mínimo de 60 jovens por organismo adulto) não se repetiu em $\mathrm{F}_{2}$, onde obteve-se uma média de 210 jovens por fêmea (Tabela 2). Já a complementação da alimentação apenas com ração digerida (RD) permitiu o atendimento aos critérios estabelecidos para reprodução de Daphnia em ambas as gerações, ainda que em $\mathrm{F}_{2}$ a média tenha sido significativamente inferior àquela obtida no tratamento com RL (Tabela 2). Na avaliação do cultivo de Ceriodaphnia dubia registrou-se, nas três gerações, uma sobrevivência insatisfatória na dieta com ração acrescida de levedura (RL), com diferença estatística significativa em relação ao outro tratamento $(\mathrm{RD})$ nas gerações $\mathrm{F}_{1}$ e $\mathrm{F}_{3}$; em $\mathrm{F}_{2}$ esta variável biológica esteve abaixo do mínimo de $80 \%$ em ambas as dietas (Figura 1b). Considerando-se a reprodução deste microcrustáceo, não se registrou diferença significativa entre as médias de jovens produzidas nos dois tratamentos; contudo, na dieta com ração digerida obteve-se um coeficiente de variação mais elevado e uma produtividade inferior a 15 jovens/adulta em $\mathrm{F}_{2}$ (Tabela 2).

\section{Efeitos da digestão da ração}

Considerando a sobrevivência de Daphnia similis, observa-se que no tratamento em teste, ou seja, ração solubilizada (RS), esta variável foi mais estável e nunca inferior a $90 \%$ nas oito gerações (Figura 2). Em contrapartida, organismos submetidos à dieta com ração digerida, apresentaram em $\mathrm{F}_{2}$ e $\mathrm{F}_{6}$ sobrevivência inferior a $80 \%$. Quanto à reprodução, em todas as gerações a $1^{\mathrm{a}}$ cria ocorreu entre o $7^{\circ}$ e o $9^{\circ}$ dia, atendendo, dessa forma, ao critério proposto em EPS1/RM/11 (ENVIRONMENT CANADA, 1990) de que a mesma deve ocorrer até o $12^{\circ}$ dia. Observa-se na tabela 3, que os organismos submetidos ao tratamento com ração digerida (RD) produziram maior número de jovens em $50 \%$ das gerações, embora apenas em duas (gerações 3 e 4), tal diferença tenha sido significativa do ponto de vista estatístico; nas demais gerações, os organismos que receberam ração solubilizada (RS) apresentaram médias de jovens mais elevadas, com diferença significativa apenas em $\mathrm{F}_{6}$. Também na tabela 3, verifica-se que para a ração solubilizada as médias de jovens foram 2 a 4 vezes superiores a 60 organismos/adulta, fato também observado na dieta com ração digerida, exceção feita à geração $\mathrm{F}_{6}$. Por outro lado, a dieta com ração solubilizada resultou em um menor coeficiente de variação da reprodução ao longo de todas as gerações mantidas $(21,8 \%$ contra $31,8 \%$ registrado no tratamento com ração digerida).

$\mathrm{Na}$ dieta com ração solubilizada, a sobrevivência de Ceriodaphnia dubia manteve-se entre 95 e $100 \%$ em todas as 


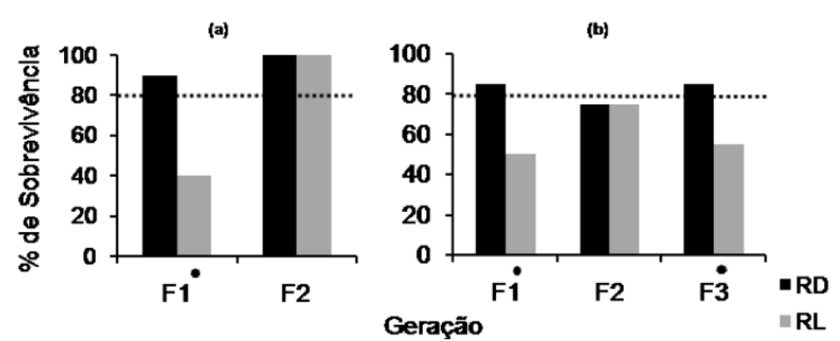

Figura 1 - Sobrevivência (em \%) de Daphnia similis (a) e Ceriodaphnia dubia (b) nos tratamentos com (RL) ou sem (RD) adição de levedura

à ração. A linha indica a porcentagem de sobrevivência mínima dos organismos adultos para que a utilização dos jovens produzidos seja viável.

(*) Geração com diferença estatisticamente significativa entre os tratamentos, com nível de significância $(\alpha)$ de 5\%.

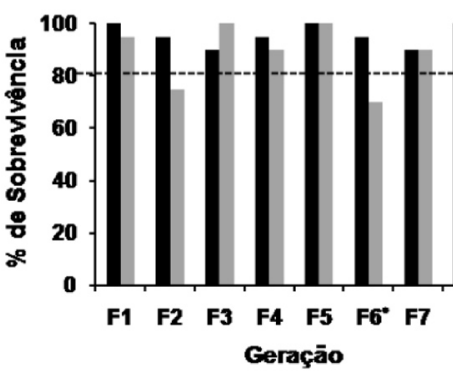

Figura 2 - Sobrevivência (\%) de Daphnia similis nas dietas Ração Solubilizada (RS) e Ração Digerida (RD). A linha indica a porcentagem de sobrevivência mínima dos organismos adultos para que a utilização dos jovens produzidos seja viável.

*Geração com diferença estatisticamente significativa entre os tratamentos, com nível de significância $(\alpha)$ de $5 \%$.

Tabela 2 - Reprodução de Daphnia similis e Ceriodaphnia dubia ( $\mathrm{N}^{\mathrm{o}}$ de jovens/fêmea) nos tratamentos com (RL) ou sem (RD) adição de levedura à ração.

\begin{tabular}{lcccc}
\hline Geração & \multicolumn{2}{c}{ Daphnia similis } & \multicolumn{2}{c}{ Ceriodaphnia dubia } \\
\cline { 2 - 5 } & $\begin{array}{c}\text { Ração }+ \\
\text { levedura } \\
38,9 \pm 66,1\end{array}$ & $\begin{array}{c}\text { Ração } \\
\text { digerida }\end{array}$ & Ração + levedura & Ração \\
& $103,1 \pm 57,6$ & $19 \pm 5,7$ & $21,5 \pm 4,8$ \\
$\mathrm{~F}_{1(*)}$ & $210 \pm 22,2$ & $152,5 \pm 20,8$ & $16,5 \pm 4,4$ & $12,0 \pm 3,3$ \\
$\mathrm{~F}_{2\left(^{*}\right)}$ & - & - & $19,0 \pm 5,7$ & $16,6 \pm 3,4$ \\
$\mathrm{~F}_{3}$ & $124,5 \pm 121$ & $127,8 \pm 34,9$ & $18,2 \pm 1,4$ & $16,7 \pm 4,8$ \\
Média & 97,2 & 27,3 & 7,7 & 28,7 \\
Coeficiente de & variação (\%) & & 7,7 & 2 \\
\hline
\end{tabular}

(*)Diferença estatisticamente significativa entre os tratamentos com nível de significância $(\alpha)$ de $5 \%$.

Tabela 3 - Reprodução de Daphnia similis ( $\mathrm{N}^{\mathrm{o}}$ de jovens/fêmea) nas dietas com Ração Solubilizada (RS) e Ração Digerida (RD).

\begin{tabular}{lcc}
\hline & \multicolumn{2}{c}{ Dieta } \\
\cline { 2 - 3 } Geração & $\begin{array}{c}\text { Suspensão algácea }+ \\
\text { Ração solubilizada (RS) }\end{array}$ & $\begin{array}{c}\text { Suspensão algácea }+ \\
\text { Ração digerida (RD) }\end{array}$ \\
\hline $\mathrm{F}_{1}$ & $242,1 \pm 51,5$ & $270,2 \pm 73,8$ \\
$\mathrm{~F}_{2}$ & $189,9 \pm 59,7$ & $178,3 \pm 99,1$ \\
$\mathrm{~F}_{3}{ }^{*}$ & $183,7 \pm 57,1$ & $243,1 \pm 18,7$ \\
$\mathrm{~F}_{4}{ }^{*}$ & $166,2 \pm 48,4$ & $253,2 \pm 67,8$ \\
$\mathrm{~F}_{5}$ & $166,6 \pm 34,9$ & $157,4 \pm 19,0$ \\
$\mathrm{~F}_{6}{ }^{*}$ & $133,5 \pm 26,6$ & $88,0 \pm 57,4$ \\
$\mathrm{~F}_{7}$ & $240,7 \pm 74,3$ & $199,4 \pm 82,5$ \\
$\mathrm{~F}_{8}$ & $249,3 \pm 26,6$ & $264,9 \pm 45,1$ \\
\hline \hline Média & $196,5 \pm 42,8$ & $205,6 \pm 62,6$ \\
Coeficiente de & 21,8 & 31,8 \\
variação (\%) & \multicolumn{2}{c}{} \\
\hline
\end{tabular}

(*)Diferença estatisticamente significativa entre os tratamentos com nível de significância $(\alpha)$ de $5 \%$.

gerações (Figuras 3a e 3b), fato que não se repetiu no tratamento com ração digerida, onde a sobrevivência foi irregular, atingindo, já no período de 0 a 7 dias, taxas inferiores a $80 \%$ nas gerações 5 e 6 (Figura 3a). Resultados semelhantes (sobrevivência das adultas inferior a $80 \%$ ) nesse tratamento foram obtidos também em $\mathrm{F}_{2}$ e $\mathrm{F}_{3}$ porém, no período de 7 a 14 dias (Figura 3b).

Ainda com relação a Ceriodaphnia dubia, no período de 0 a 7 dias, o tratamento com ração digerida (RD) promoveu uma reprodução mais elevada em $75 \%$ das 8 gerações, embora a análise estatística tenha apontado tal diferença como significativa apenas em $\mathrm{F}_{4}$ e $\mathrm{F}_{5}$ (Tabela 4). Nas gerações $\mathrm{F}_{2}$ e $\mathrm{F}_{7}$, a dieta com ração solubilizada (RS) promoveu um melhor desempenho (embora não significativo sob o aspecto estatístico), permitindo sempre a obtenção de médias de jovens superiores ao mínimo (15). No período de 7-14 dias, as diferenças tornaram-se ainda menores, exceção feita à geração 5 , em que a média de jovens resultante no tratamento com ração digerida foi expressivamente inferior, em função da elevada mortalidade das adultas. Ainda na tabela 4, verificase que o coeficiente de variação da reprodução ao longo das gerações foi inferior no tratamento com ração solubilizada, em ambos os períodos considerados.

Estudos desenvolvidos nas décadas de 80 e 90 (Cowgill et al., 1985; Lee et al., 1986; Norberg-King \& Schmidt, 1993), demonstram que, quanto maior o valor nutricional do alimento, maior a sobrevivência e a reprodução dos organismos. Assim, a dieta dos dafinídeos pode incluir, além de uma espécie de alga, outro complemento nutricional, entre os quais bactérias e protozoários (Goulden et al., 1982), cujo crescimento é estimulado por compostos como o RL, ou seja, ração para peixes digerida e levedura. Beatrici et al. (2006) verificaram que a inclusão desse complemento à dieta constituída de suspensão algácea, dobrou a produção diária de jovens de Daphnia similis, quando cultivada em meio básico.

Particularmente, Ceriodaphnia dubia é um "consumidor de bactérias", de modo que dietas constituídas somente por algas não sustentam uma boa reprodução e sobrevivência das culturas (Cooney et al., 1992; Norberg \& Mount, 1984). Cooney et al., (1992), em experimentos testando 8 dietas para 
Ceriodaphnia dubia, obtiveram resultados semelhantes com as constituídas por Pseudokirchneriella subcapitata mais ração de truta solubilizada e Pseudokirchneriella subcapitata mais ração de truta solubilizada, levedura e Cerophyl®. Também em 1992, Knight \& Waller verificaram que a inclusão de ração de truta solubilizada no alimento composto fornecido às culturas de Ceriodaphnia dubia, permitiu a obtenção de 30,0 jovens por adulta, média muito próxima da registrada quando utilizada ração digerida no referido alimento (31,3 jovens/adulta). Dessa forma, concluíram que a dieta

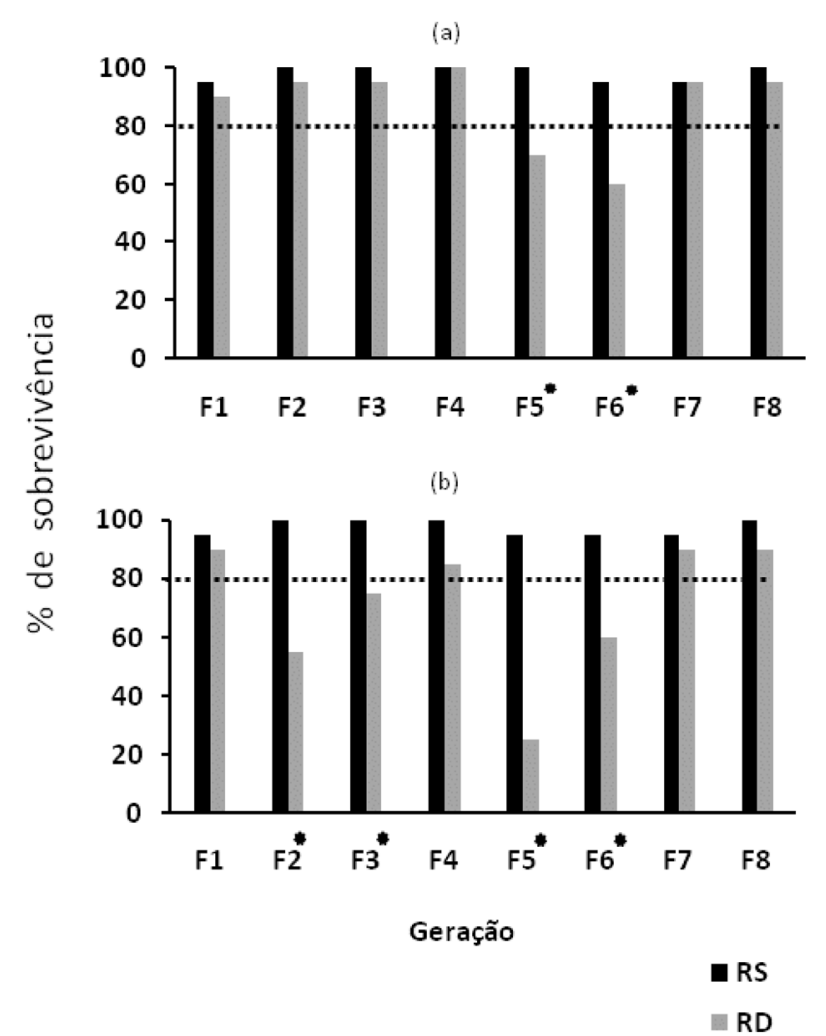

Figura 3 - Sobrevivência de Ceriodaphnia dubia nas dietas Ração Solubilizada (RS) e Ração Digerida (RD): (a) período de 0 a 7 dias; (b) período de 7-14 dias. A linha indica a porcentagem de sobrevivência mínima dos organismos adultos para que a utilização dos jovens produzidos seja viável.

*Geração com diferença estatisticamente significativa entre os tratamentos, com nível de significância $(\alpha)$ de $5 \%$. constituída de suspensão de Pseudokirchneriella subcapitata e ração solubilizada poderia proporcionar níveis aceitáveis de sobrevivência e reprodução.

Resultados semelhantes foram encontrados no trabalho de Bailey et al. (2000), envolvendo o fornecimento de ração solubilizada como alimento a Ceriodaphnia dubia. Embora tenham utilizado um procedimento distinto para o preparo dessa ração, tais autores verificaram que, em conjunto com a alga Pseudokirchneriella subcapitata esse alimento proporcionou porcentagens de sobrevivência entre 80 e $100 \%$ dos organismos adultos e produção de 25,6 a 36,5 jovens por fêmea, conforme o tipo de água utilizado no cultivo.

No presente estudo, observou-se que a complementação da dieta apenas com ração solubilizada e sem levedura permitiu o atendimento aos critérios de qualidade das culturas de Daphnia similis e Ceriodaphnia dubia, estabelecidos por procedimentos padronizados em que o complemento nutricional recomendado inclui levedura, ração digerida e Cerophyl ${ }^{\circledR}$ em iguais proporções (ENVIRONMENT CANADA, 1990; 1992; USEPA, 2002a; 2002b). Além disso, possivelmente por apresentar uma composição mais constante, a dieta com ração solubilizada apresentou menores coeficientes de variação para a reprodução ao longo das gerações, considerando ambos os microcrustáceos.

Portanto, a solução de ração solubilizada e sem levedura, preparada conforme descrito neste estudo pode ser utilizada como alimento complementar das culturas de Daphnia similis e Ceriodaphnia dubia, sustentando adequadamente a sobrevivência e reprodução desses organismos e minimizando sua variabilidade.

\section{REFERÊNCIAS}

ABNT, Associação Brasileira de Normas Técnicas, 2004, NBR 12713. Ecotoxicologia Aquática - Toxicidade aguda - Método de ensaio com Daphnia spp.(Crustacea, Cladocera). Rio de Janeiro. 21 p.

ABNT, Associação Brasileira de Normas Técnicas, 2005, NBR 13373. Ecotoxicologia Aquática - Toxicidade crônica - Método de ensaio com Ceriodaphnia spp. (Crustacea, Cladocera). Rio

Tabela 4 - Reprodução de Ceriodaphnia dubia ( ${ }^{\circ}$ de jovens/fêmea) nas dietas Ração Solubilizada (RS) e Ração Digerida (RD).

\begin{tabular}{lcccc}
\hline \multirow{2}{*}{ Geração } & \multicolumn{2}{c}{ Período 0-7 dias } & \multicolumn{2}{c}{ Período 7-14 dias } \\
\cline { 2 - 5 } & $\begin{array}{c}\text { Ração } \\
\text { solubilizada }\end{array}$ & Ração digerida & $\begin{array}{c}\text { Ração } \\
\text { solubilizada }\end{array}$ & $\begin{array}{c}\text { Ração } \\
\text { digerida }\end{array}$ \\
\hline $\mathrm{F}_{1}$ & $22,4 \pm 9,9$ & $28,2 \pm 12,5$ & $50,4 \pm 15,6$ & $58,4 \pm 14,1$ \\
$\mathrm{~F}_{2}$ & $26,8 \pm 6,5$ & $21,4 \pm 8,1$ & $42,6 \pm 11,8$ & $45,8 \pm 25,8$ \\
$\mathrm{~F}_{3}$ & $25,2 \pm 5,3$ & $26,1 \pm 7,3$ & $43,1 \pm 12,9$ & $48,2 \pm 15,4$ \\
$\left.\mathrm{~F}_{4}{ }^{*}\right)$ & $21,2 \pm 7,2$ & $38,6 \pm 19,3$ & $45,5 \pm 19,7$ & $60,1 \pm 24,8$ \\
$\mathrm{~F}_{5}^{\left({ }^{*}\right)}$ & $24,5 \pm 5,8$ & $30,9 \pm 12,2$ & $47,2 \pm 15,6$ & $26,5 \pm 13,2$ \\
$\mathrm{~F}_{6}$ & $26,5 \pm 4,6$ & $28,6 \pm 5,3$ & $56,6 \pm 15,8$ & $51,0 \pm 12,6$ \\
$\mathrm{~F}_{7}$ & $28,4 \pm 9,2$ & $27,5 \pm 7,2$ & $62,4 \pm 9,9$ & $60,9 \pm 13,5$ \\
$\mathrm{~F}_{8}$ & $19,5 \pm 4,9$ & $20,6 \pm 11,8$ & $55,9 \pm 7,7$ & $48,1 \pm 24,2$ \\
\hline Média geral & $24,3 \pm 3,1$ & $27,7 \pm 5,6$ & $50,5 \pm 7,2$ & $49,9 \pm 11,1$ \\
Coeficiente de & 12,6 & 20,3 & 14,2 & 22,3 \\
variação (\%) & & & & \\
\hline
\end{tabular}

(*)Diferença estatisticamente significativa entre os tratamentos com nível de significância $(\alpha)$ de $5 \%$. 
de Janeiro. 15p.

ASTM, American Society for Testing and Materials 1997. E119397. Standard guide for conducting Daphnia magna life-cycle toxicity tests. $18 \mathrm{p}$.

BAILEY, H.C.; KRASSOI, R.; ELPHICK, J.R.; MULHALL, A.M.; HUNT, P.; TEDMANSON, L.; LOVELL, A, 2000, Application of Ceriodaphnia dubia for whole effluent toxicity tests in the Hawkesbury - Nepean Watershed, New South Wales, Australia: method development and validation. Environ Toxicol Chem, 19 (1): 88-93.doi: 10.1002/etc.5620190110.

BAIRD, D. J.; BARBER, I.; BRADLEY, M.; CALOW, P.; SOARES, A. M. V. M., 1989, The Daphnia bioassay: a critique. Hydrobiol, 188/189: 403-406. doi: 10.1007/BF00027806.

BEATRICI, A. C.; ARENZON, A.; COIMBRA, N. J.; RAYA RODRIGUEZ, M. T., Fertilidade e sensibilidade de Daphnia similis e Daphnia magna submetidas a diferentes cultivos, 2006, J Braz Soc Ecotoxicol, 1(2): 123-126. doi: 10.5132/ jbse.2006.02.006.

BRADLEY, M.C.; NAYLOR, C.; CALOW, P.; BAIRD, D.J.; BARBER, I.; SOARES, A.M.V.M., 1993, Reducing variability in Daphnia toxicity tests - a case for further standardization. In: Progress in Standardization of Aquatic Toxicity Tests. Soares, A.M.V.M. \& Calow, P. eds. Lewis Publishers, Michigan. 208 p.

COONEY, J.D.; DE GRAEVE, G.M.; MOORE, E.L.; PALMER, W.D. \& POLLOCK, T.L., 1992, Effects of food and water quality on culturing of Ceriodaphnia dubia. Environ Toxicol Chem, 11(6): 823-837. doi: 10.1002/etc.5620110611.

COWGILL, U. M.; KEATING, K.I. and TAKAHASHI, I. T., 1985, Fecundity and longevity of Ceriodaphnia dubia/affinis in relation to diet at two different temperatures. J Crust Biol, 5, 420-429.

ENVIRONMENT CANADA, 1990, Biological test method: Acute lethality test using Daphnia spp. Environmental Protection Series. Report EPS 1/RM/11. p.

ENVIRONMENT CANADA, 1992, Biological test method: Test of reproduction and survival using the cladoceran Ceriodaphnia dubia. Environmental Protection Series. Report EPS 1/RM/21- 72p.

GOULDEN, C.E.; COMOTTO, R.M.; HENDRICKSON JR, J.A.; HORNING, L.L.; JOHNSON, K.L., 1982, Procedures and recommendations for the culture and use of Daphnia in bioassay studies.In: Aquatic Toxicology and hazard Assessment: Fifth
Conference. ASTM STP 766 Eds: J.G. Pearson; R.B. Foster and W.E. Bishop. American Society for Testing and Materials. p 139-160.

ISO- International Standardization Organization (2000). Water quality - Determination of long-term toxicity of substances to Daphnia magna Straus (Cladocera, Crustacea). ISO 10706. 17p.

KEATING, K. I., 1985, A system of defined (sensu strictu) media for Daphnid (Cladocera) culture. Wat Res, 19: 73-78. doi: 10.1016/0043-1354(85)90325-2.

KNIGHT, J. T. \& WALLER, W. T., 1992, Influence of the addition of Cerophyl on the Selenastrum capricornutum diet of the cladoceran Ceriodaphnia dubia. Environ Toxicol Chem, 11(4): 521-534. doi: 10.1002/etc.5620110410.

LA ROCCA, C.A.; FRANCISCO, D.E.; DI GIANO, F.A., 1994, Effects of diet on survival, reproduction and sensitivity of Ceriodaphnia dubia . Wat Environ Res 66(7): 905-911. doi:10.2175/WER.66.7.7.

LEE, C.M.; TURNER, C.A.; HUNTINGTON, E., 1986, Factors affecting the culture of Daphnia magna. In: Aquatic Toxicology and Environmental Fate: Ninth Volume, ASTM STP 921 . Eds. T.M. Poston and R. Purdy. American Society for Testing and Materials, Philadelphia, p. 357-368.

NORBERG, T.J. \& MOUNT, D.I., 1984, Diets for Ceriodaphnia reticulata life cycle tests. In: Cardwell, R.D.; Purdy, R. and Bahmer, R.C. eds. Aquatic Toxicology and Hazard Assessment 7 th. Symposium ASTM STP 854. American Society for Testing and Materials, Philadelphia p. 42-52.

NORBERG-KING, T. J. and SCHMIDT, S., 1993, Comparison of effluent toxicity results using C. dubia cultured on several diets. Environ Toxicol Chem 12(10): 1945-1955. doi: 10.1002/ etc. 5620121020 .

USEPA.,2002a, Short-term methods for estimating the chronic toxicity of effluents and receiving waters to freshwater organisms. Fourth edition. Washington, D.C. (EPA-821-R-02-13). 335 p.

USEPA., 2002b, Methods for measuring the acute toxicity of effluents and receiving waters to freshwater and marine organisms. Fifth edition. Washington, D.C. (EPA-821-R-02-012). 266p.

WEST, INC. \& GULLEY, D., 1996, Toxstat Version 3.5 - University of Wyoming. 